\title{
SENTIMENTAL ANALYSIS USING CONVOLUTIONAL NEURAL NETWORK
}

\author{
Namratha Bhat \\ Department of ISE \\ JSS Academy of Technical Education \\ Bangalore, India
}

\begin{abstract}
As we see rapid growth in the field of opinion mining, there is high scope for Sentimental Analysis, which effectively identifies and extracts subjective information. Sentimental Analysis is the one, which uses Natural Language Processing to successfully classify the expressed feelings in diverse manners such as positive, negative or neutral. It aims at discovering text or opinions present on social media platform along with the calculation of polarity. As sentiments of customers are very essential for the growth of the company, data which is unstructured or incomplete, need to be properly managed and this is where Sentimental Analysis Plays an important role. In order to solve all these problems, Sentimental Analysis is combined with Deep Learning, as DL models are well known for their high performance.
\end{abstract}

Keywords- CNN, Dl, NLP, Neural Network Pre-Processing, SA, Sentiment

\section{INTRODUCTION}

\section{A. Sentimental Analysis:}

Sentimental Analysis can be defined as classification and interpretation of the emotions in text data with the help of text analysis techniques. It helps businesses in identifying their customer's sentiment toward products and services in online feedback and conversations. As studying thousands of reviews is time consuming and hectic, some method should be used to do this work automatically and easily. This is where Sentimental Analysis comes into picture. It is the subclass of Natural Language processing. Analysis of sentiment is the method of extracting knowledge from user's opinion. Each Individual shares opinion on social media, which is to be detected and analyzed.

The rest of the paper is organized as follows. Proposed embedding and extraction algorithms are explained in section II. Experimental results are presented in section III. Concluding remarks are given in section IV.

\author{
Dr. Nethravathi B \\ Department of ISE \\ JSS Academy of Technical Education \\ Bangalore, India
}

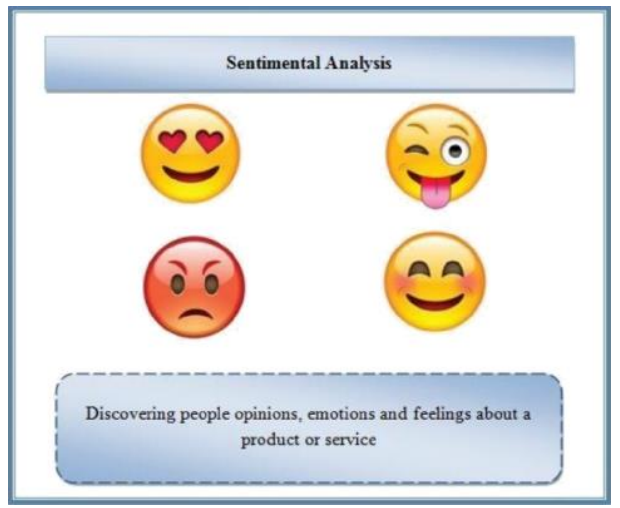

Fig 1. Sentimental Analysis.

Some of the most popular types of Sentimental Analysis (SA) are

1. Fine-grained SA:

If we want polarity precision in our business, we can expand our polarity categories to include:

- Highly positive

- Positive

- Neutral

- Negative

- Highly Negative

This is what Fine-grained SA is. This type of SA can be used to detect emotions such as happiness, sadness, anger etc.

2. Aspect-based SA: Sometimes while analyzing sentiment of texts, we may want to know which particular feature customers are mentioning. This is where Aspect-based SA can help. For example, if a review says, "The camera quality of this phone is not good", an aspect-based classifier can tell that this particular customer is expressing negative opinion about camera of that particular phone. 3. Multilingual SA: This process is quite difficult as it involves lot of pre-processing and resources which are either available online or have to be created manually.

B. How does Sentimental Analysis work?

Sentimental Analysis makes use of different NLP methods and algorithms such as,

- $\quad$ Rule Based 
- Automatic

- Hybrid

1. Rule-Based:

This system uses set of rules created by language experts to classify text as positive, negative or neutral. It may include some of the techniques like Stemming, tokenization, parsing and lexicons.

* In the first step of the process, it defines two lists one with positive and another with negative words.

* Then in second step, number of positive and negative words that appear in given text is counted.

* Then it returns positive sentiment if the appearance of positive words is more and, vice versa.

2. Automatic approaches: This is the one, which uses Machine Learning Techniques and not manually created rules. Classification step includes models such as Logistic Regression, support vector machine, Deep learning models etc. 3. Hybrid approaches: They combine features of rule based and automatic approaches into one-system and give results, which are more accurate.

\section{Deep Learning (DL):}

Deep Learning, which acts as a function of Artificial Intelligence comes into scope with the idea of mimicking human brain. DL is a subgroup of machine learning. As ML algorithms cannot deal with high dimensional data and has problem in feature extraction, DL model can help us solve those problems. DL has a network that can learn unsupervised even from unstructured or incomplete data. Some of the DL models are Convolutional Neural Network(CNN), Recurrent Neural Network(RNN), Deep Neural Network etc.

The one we are going to discuss about in this paper is:

\section{Convolutional Neural Network (CNN):}

CNNs are the form of Neural Networks with convolutional layers which may be one or more in number and are used for the tasks like image processing, classification, segmentation etc. Convolution is the one which slides over the input. CNN's are made up of several layers of convolutions that comes with ReLU or Tanh(Activation functions) which is applied to the outcomes. Contrast to previous feed-forward neural network where each input neuron is connected to each output neuron in the following layer, in CNNs convolutions are used above the input layer to complete the output. Each layer combines the result by applying several thousands of different filters.

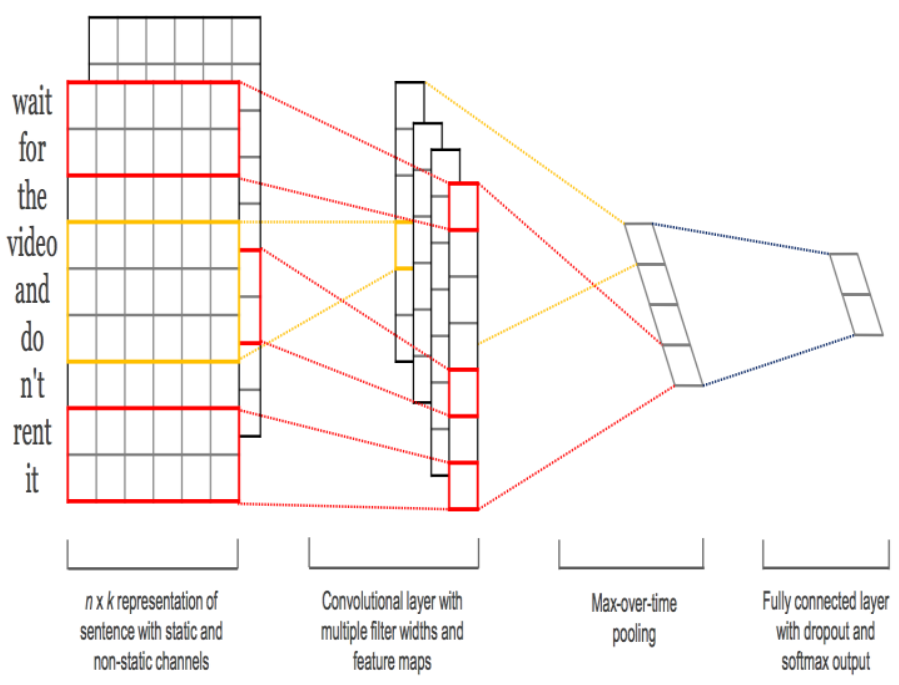

Fig 2. Convolutional Neural Network.

Architecture of CNN: CNN includes input, output and also some hidden layers which encompasses a chain of convolutional layers which convolve over input. While we are dealing with $\mathbf{C N N}$, input is in the form of tensor with shape (product of (number of images), (image width), (image height), (image depth)). Which after passing through a convolutional layer, becomes abstracted to a feature map, with shape (product of (number of images), (feature map width), (feature map height), (feature map channels).Convolutional layer must contain following attributes:

- Convolutional kernels with their width and height

- The number of input channels and output channels.

- Depth of Convolution filter (Input channels) which should be same as number channels (depth) of the input feature map.

Convolution layer convolve through the given input and pass it to the subsequent layer

Pooling Layer: Convolutional networks may contain local or global pooling layers to facilitate the principal estimation. Pooling layer combines output of neuron clusters from every layer into single neuron in following layer thereby reducing size of data. In addition, pooling layer can also be used to calculate a max or an average. Max pooling considers the extreme value from each of a bunch of neurons at the previous layer. 


\section{International Journal of Engineering Applied Sciences and Technology, 2020 Vol. 5, Issue 3, ISSN No. 2455-2143, Pages 422-426 \\ Published Online July 2020 in IJEAST (http://www.ijeast.com)}

Fully connected: Fully connected layers are used to connect every neuron of one layer to every neuron of following layer. The flattened matrix gets on to the fully connected layer to categorize the input.

\section{LITERATURE SURVEY}

Author in the paper [1] explains how Local-scale Convolutional neural network can be used to perform sentimental analysis. $\mathrm{CNN}$ with data augmentation technology is used to analyze user satisfaction on a product, happiness with a particular environment or destructive situation after disaster. This model outperformed most of the modern models and higher accuracy performance in classification of Twitter sentiment could be seen. However, the disadvantage was this model could only employ smaller training dataset. In the paper [2] where the likely hood of the movie reviews is analyzed, author has explained the use of DRI- RCNN (Deceptive Review identification by RCNN) and results of which outlined that the design outperformed LSTM by $1-2 \%$ in terms of accuracy. In the paper [3], sentimental analysis using deep learning is integrated with Bag-of- words and is utilized in analyzing public view on various companies using Facebook data. The proposed model achieved $3.36 \%$ test accuracy, but the disadvantage was it utilized smaller training dataset and needed high level preprocessing in order to get high accuracy. In the paper [4], which focuses on analyzing sentiments from corporate surveys like google opinions to movie reviews, Recurrent Neural Network (RNN) is used along with feature extraction using unigrams and bigrams. The model could be used to carry out SA on various class of text. Nevertheless, as it tends to classify the different level of texts, ambiguous words present in the document can hamper the accuracy on the whole.

\section{METHEDOLOGY}

Data available in the world most of the time is either unstructured or incomplete and thus becomes difficult for computers to comprehend and extract data. Natural Language Processing(NLP) is the subset of Artificial Intelligence that aims at making computers understand and process human languages. We need to follow certain steps before feeding data into the classifier:

1. Sentence Segmentation: here the text is broken into separate sentences.

2. Word Tokenization: now each sentence is broken into words which are also called tokens.

3. Predicting Parts of Speech for Each Token: now each token is to be analyzed to guess its parts of speech. This can be achieved by feeding each token into separate created model for detecting parts of speech.

4. Text Lemmatization: here the same words with different forms are found so that later we'll know that both the sentences are talking about the same concept. This process called Lemmatization can be achieved with the help of look up table of lemma forms of words.

5. Identifying Stop Words: here we identify and remove words that appear more often such as "and", "the" and "a".

6. Dependency Parsing: this is used to understand how different words in the sentences are related to one another. Here we build a tree where one parent word is allocated to other words in a sentence.

7. Named Entity Recognition: here the nouns in the sentence are detected and labelled with real-world concepts they represent.

8. Conference Resolution: after all this, there is still one more problem that is to be addressed. Pronouns are the shortcuts, which are used in the place of nouns. Hence, conference resolution aims at tracking these pronouns.

After the pre-processing of data is done, it should be fed to CNN classifier. Before sending it to the classifier we need to build a vocabulary which helps in converting each token into integer numbers that is, we create word embedding. Here we are transforming each word into a set of numbers. Now the document is represented in the form of matrix where each row of the matrix holds a vector that represents a word. And this matrix is fed to Convolutional layer of $\mathbf{C N N}$ where filters slide over complete rows of the matrix. We consider a block from input matrix, multiply each of the cell with corresponding weights and copy the weighted sum to the output matrix. And then we move the block by one and continue the same process. Later in the pooling layer, we apply max function to the result of each filter.one important reason for using pooling layer is, it outputs a fixed-sized matrix. Pooling can also work on the output dimensionality problem. Then output is finally transferred to a fully connected layer, which contains dropout and soft max layers. Dropout layer helps in regularizing the convolutional neural network. Finally, softmax layer classifies the text into positive or negative.

\section{Consider for example figure 3 ,}

Here we illustrate 3 filter region sizes where each region, in turn, has 2 filters. Here each filter executes convolution on sentence matrix thereby generating feature maps. Following which 1 max-pooling layer is applied to each map recording largest number from each feature map.

Thus, we get univariate feature vector from total six maps, and these 6 features are added and a feature vector for the ultimate layer is formed. Finally, this feature vector is fed as an input to soft max layer, which uses it to classify sentence. 


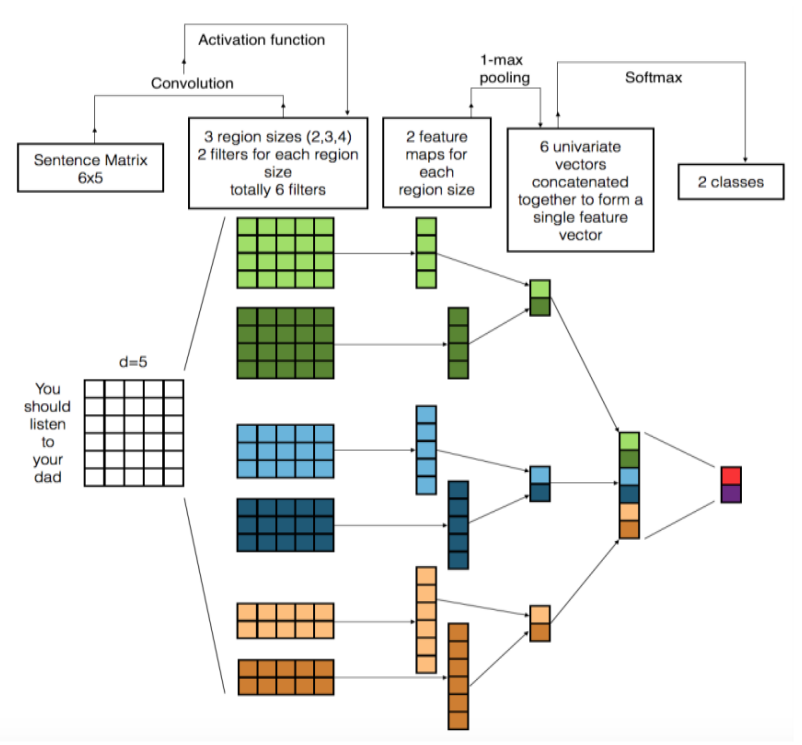

Fig 3. Example showing how CNN classify text

\section{CONCLUSION}

Social media is an important platform for people to express their sentiments. Which results in a huge amount of incomplete and unstructured data. Business organizations need to analyse these sentiments to attain business insights. This is where sentimental analysis comes into picture. Rapidly spreading hate speech on social media is to be detected efficiently in order to remove the misunderstandings and conflicts going on between people.

Lately, DL algorithms provided remarkable performance in the field of NLP encompassing SA across different datasets. Such models actually learn required features on their own.

\section{ACKNOWLEDGEMENT}

The author in the paper here would like to thank their organization JSS Academy of Technical Education for providing this wonderful opportunity to carry out this task.

\section{REFERENCES}

[1] Garain Avishek. \& Mahata Sainik Kumar. (2019). Sentiment Analysis at SEPLN Sentiment Analysis at Tweet level using Deep Learning.arXiv:1908.00321v1 [cs.CL] 1Aug 2019.

[2]A Kalaivani \& D Thenmozhi. (2019). Sentiment Analysis using Deep Learning Techniques: A survey on Sentiment Analysis using Deep Learning Techniques. International Journal of Recent Technology and Engineering(IJRTE) ISSN:2277-3878, Volume-7, Issue-6s5,April 2019.

[3] Charoenkwan Phasit. (2018). ThaiFBDeep: A Sentimental Analysis using Deep Learning combined with Bag-of-Words features on Thai Facebook Data. DOI 10.1109/IIAIAAI.2018.00120.

[4] Kaur Jaspreet \& Kaur Sidhu Brahmaleen. (2018). Sentiment Analysis Based on Deep Learning Approaches. IEEE Xplore Compliant Part Number: CFP18K74-ART; ISBN:978-1-5386-2842-3.

[5] Souma, W., Vodenska, I. \&Aoyama, H. Enhanced news sentiment analysis using deep learning methods. jComput Soc 2, 33-46(2019).

https://doi.org/10.1007/s42001-019-00035-X.

[6] M. I. Prabha and G. Umarani Srikanth, "Survey of Sentiment Analysis Using Deep Learning Techniques," 2019 1st International Conference on Innovations in Information and Communication Technology (ICIICT), CHENNAI, India, 2019, pp. 1-9, doi: 10.1109/ICIICT1.2019.8741438.

[7] Tsantekidis, A., Passalis, N., Tefas, A., Kanniainen, J., Gabbouj, M., \& Iosifidis, A. (2017). Fore- casting stock prices from the limit order book using convolutional neural

networks. In: Business informatics (CBI), 2017 IEEE 19th conference on. vol. 1, pp. 7-12. IEEE.

[8] Nhan Cach Dang., María N. Moreno-García \&Fernando De la Prieta.(2020). Sentiment Analysis Based on Deep Learning: A Comparative Study. Electronics 2020, 9, 483; doi:10.3390/electronics9030483.

[9] Khurana, Diksha \& Koli, Aditya \& Khatter, Kiran \& Singh, Sukhdev. (2017). Natural Language Processing: State of The Art, Current Trends and Challenges.

[10] S. Albawi, T. A. Mohammed and S. Al-Zawi, "Understanding of a convolutional neural network," 2017 International Conference on Engineering and Technology (ICET), Antalya, 2017, pp. 1-6, doi: 10.1109/ICEngTechnol.2017.8308186.

[11] Yamashita, R., Nishio, M., Do, R.K.G. et al. Convolutional neural networks: an overview and application in radiology. Insights Imaging 9, 611-629 (2018). https://doi.org/10.1007/s13244-018-0639-9.

[12] Talib Ramzan., Kashif Hanif Muhammad.,Ayesha Shaeela \& Fakeeha Fatima.(2016). Text Mining: Techniques, Applications and Issues. (IJACSA) International Journal of Advanced Computer Science and Applications, Vol. 7 No. 11, 2016.

[13]Al-Hassan, Areej \& Al-Dossari, Hmood. (2019). DETECTION OF HATE SPEECH IN SOCIAL NETWORKS: A SURVEY ON MULTILINGUAL CORPUS. 83-100. 10.5121/csit.2019.90208.

[14] Mika V. Mäntylä, Daniel Graziotin, Miikka Kuutila, The evolution of sentiment analysis-A review of research topics, venues, and top cited papers, Computer Science Review, Volume 27, February 2018, Pages 16-32, ISSN 1574-0137, https://doi.org/10.1016/j.cosrev.2017.10.002.

(https://www.sciencedirect.com/science/article/pii/S15740137 17300606).

[15]Sohangir, S., Wang, D., Pomeranets, A., \& Khoshgoftaar, T. M. (2018). Big data: deep learning for financial sentiment analysis. Journal of Big Data, 5(1), 3. 
https://doi.org/10.1186/s4053 7-017-0111-6.

[16] Xing, F. Z., Cambria, E., \& Welsch, R. E. (2018). Natural language based financial forecasting: a survey. Artificial Intelligence Review, 50(1), 49-73.

[17] Troiano, L., Mejuto, E., \& Kriplani, P. (2017). On feature reduction using deep learning for trend prediction in finance. arXiv preprint arXiv: 1704.03205

[18] Tsantekidis, A., Passalis, N., Tefas, A., Kanniainen, J., Gabbouj, M., \& Iosifidis, A. (2017). Using deep learning to detect price change indications in financial markets. In: Signal processing confer-ence (EUSIPCO), 2017 25th European. pp. 2511-2515. IEEE. 